\title{
Ibuprofen verhindert Höhenkrankheit
}

\author{
Wenn Sie eine Trekkingtour im Hima- \\ laja planen, lohnt es sich vielleicht, \\ etwas Ibuprofen mit in den Rucksack \\ zu packen.
}

- Die akute Höhenkrankheit ist eine Symptomenkonstellation aus Kopfschmerzen, Schlafstörungen, Müdigkeit, Schwindel, Übelkeit, Erbrechen und Appetitlosigkeit, die üblicherweise beim raschen Aufstieg in Höhen über $2500 \mathrm{~m}$ auftritt. Die Inzidenz hängt im Wesentlichen von der Höhe und vor allem von der Geschwindigkeit des Aufstiegs ab und erreicht bei Touristen, Trekkern und Bergsteigern Werte zwischen 25 und $75 \%$. Die Symptomatik stellt sich üblicherweise 6-12 h nach Erreichen der Höhe ein. In den meisten Fällen ist die Erkrankung selbst limitiert, kann aber bei entsprechendem Schweregrad den Betroffenen zu völliger Inaktivität verurteilen und in Einzelfällen durch ein Höhenhirnödem zum Tode führen.

Der einfachste und sicherste Weg, die Höhenkrankheit zu vermeiden, wäre ein langsamer Aufstieg mit längeren Verweildauern auf verschiedenen Höhenniveaus. Dieses Verfahren ist aber bei vielen Touristen aus Zeitmangel, bei Rettungsteams und Soldaten durch die Natur des Auftrags nicht möglich. Die genaue Pathophysiologie der akuten Höhenkrankheit ist unbekannt, doch dürften eine Vasodilatation der zerebralen Gefäße, entzündliche Prozesse und eine Schrankenstörung der Bluthirnschranke eine Rolle spielen. Die mögliche Beteiligung von inflammatorischen Mediatoren führte zur Therapie mit Glukokortikoiden und nicht steroidalen Antirheumatika.

In einer prospektiven, doppelblind geführten und randomisierten Studie hat man 86 gesunde Freiwillige mit dreimal $600 \mathrm{mg}$ Ibuprofen bzw. Placebo behandelt. Die Therapie begann sechs Stunden vor dem Aufstieg aus einer Höhe von $1240 \mathrm{~m}$ auf $3810 \mathrm{~m}$ und fand in den White Mountains in Kalifornien im

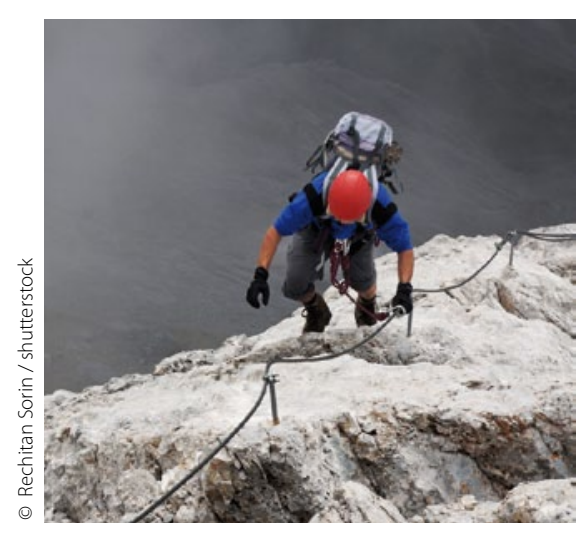

Ob Höhenkrankheit droht, hängt auch von der Aufstiegsgeschwindigkeit ab.

Juli und August 2010 statt. Wichtigste Studienziele waren das Auftreten der akuten Höhenkrankheit und ihr Schweregrad, der nach dem Score anhand des Lake Louise Questionnaire quantifiziert wurde. 44 Probanden erhielten Ibuprofen, 42 Placebo. In der Ibuprofen-Gruppe entwickelten $43 \%$ eine akute Höhenkrankheit im Vergleich zu 69\% unter Placebo. Der Schweregrad erreichte in der Placebogruppe einen Score von 4,4, unter Ibuprofen dagegen nur 3,2. Aus den Daten errechnet sich eine NNT von nur 3,9.

\section{Kommentar}

Ibuprofen erwies sich als sicheres und sehr effektives Mittel gegen die Höhenkrankheit. Gerade das Sicherheitsprofil macht Ibuprofen attraktiver als zum Beispiel Dexamethason. Eine weitere Alternative, Azetazolamid, hat den Nachteil, dass mindestens einen Tag vor dem Anstieg in die Höhe eingenommen werden soll. Leider macht die Studie keine Aussage zur notwendigen Dauer der Ibuprofen-Gabe beim längeren Aufenthalt in großen Höhen.

H. S. FÜESSL =

- G. S. Lipman et al.

(Korres.: Grant S. Lipman, MD, grant lip@hotmail.com): Ibuprofen prevents altitude illness: a randomized controlled trial for prevention of altitude illness with nonsteroidal antiinflammatories. Ann Emergency Med 2012; doi: 10.1016/j.annemergmed.2012.01.019

\section{Achtung:}

\section{Hier muss der Dummy durch eine Anzeige ersetzt werden !!}

